\title{
TOPOLOGICAL GROUPS WITH EQUAL LEFT AND RIGHT UNIFORMITIES ${ }^{1}$
}

\section{R. W. BAGLEY AND T. S. WU}

If $(G, \sigma)$ is a topological group with topology $\sigma$, there is a natural topology $\tau$ on $G$ such that $\tau \supset \sigma,(G, \tau)$ is a topological group with equal left and right uniformities and $\tau$ is the smallest such topology. The topology $\tau$ can be obtained as follows. If $V$ is a neighborhood base at the identity in $(G, \sigma)=G$, then $\left\{\bigcap_{t \in G} t V t^{-1} \mid V \in \mathcal{V}\right\}$ is a neighborhood base at the identity in $(G, \tau)=G^{*}$. Here we are interested in determining the nature of $G^{*}$ given $G$ and also in obtaining results on groups with equal left and right uniformities. For results on such groups we point to Braconnier [1] in which it is shown that a locally compact group with equal left and right uniformities has a two sided invariant Haar measure, and to N. Rickert's unpublished theorems on the structure of locally compact groups with equal uniformities. In contrast, T. S. Wu [10] has shown that left almost periodic functions on locally compact groups need not be right almost periodic. Of course, the groups here must have distinct left and right uniformities. These groups were discovered at an intermediate stage in the construction of a locally compact group $G$ for which $G^{*}$ is not locally compact. This example will be described later.

One of our principal results (Theorem 1) is that $G^{*}$ is a Lie group if $G$ is a connected Lie group. Since this depends only on the fact that $G^{*}$ is locally compact, we have attempted to determine more precisely when this occurs. As mentioned above, there are locally compact groups $G$ for which $G^{*}$ is not locally compact; however, using the result above on Lie groups we show that, if $G$ is locally compact and connected, then $G^{*}$ is locally compact. It is also shown that if a connected locally compact group $G$ with equal left and right uniformities contains a one parameter subgroup $\phi(R)$ as a normal subgroup, then $\phi(R)$ is contained in the center of $G$. The hypothesis on the uniformities is not necessary if $G / \bar{\phi}(R)$ is compact. These are Corollaries 1 and 2 of Theorem 3 .

As a preliminary we obtain some lemmas on the topology of a subgroup $H \subset G$ relative to $G^{*}$.

LEMMA 1. If $K$ is a compact normal subgroup of the connected group $G$, then the topologies of $K$ relative to $G$ and $G^{*}$ are equal.

Received by the editors January 17, 1966.

1 This work was supported by NASA Grant NGR 10-007-005. 
Proof. Let $A(K)$ be the group of all continuous automorphisms of $K$ and $I(K)$ the group of inner automorphisms of $K$. Define $\Delta: G$ $\rightarrow A(K)$ as follows: $\Delta(g)(k)=g k g^{-1}$. We note that $\Delta$ is continuous when $A(K)$ has the compact-open = uniform topology. Thus $\Delta(G)$ is a connected subgroup of $A(K)$. It follows that $\Delta(G)=I(K)$ since $A(K) / I(K)$ is totally disconnected by Theorem 1 of [6]. Let $U$ be a neighborhood of the identity in $G$. By compactness of $K$ we pick a neighborhood $V$ such that $V \subset \bigcap_{k \in K} k U k^{-1}$. We have,

$$
K \cap\left(\bigcap_{t \in G} t U t^{-1}\right)=\bigcap_{t \in G} t(U \cap K) t^{-1}=\bigcap_{k \in K} k(U \cap K) k^{-1} \supset V \cap K .
$$

This proves the lemma.

Lemma 2. If $H$ is a subgroup of $G$ such that the coset space $G / H$ is compact, then the sets $\bigcap_{h \in H} h V h^{-1}$, where $V$ is a neighborhood of $e$ in $G$, form a neighborhood base at e in $G^{*}$.

Proof. Let $V$ be a neighborhood of $e$ in $G$ and let $W$ be a symmetric neighborhood of $e$ such that $W^{3} \subset V$. Since $G / H$ is compact, $G$ $=\bigcup_{i=1}^{n} W g_{i} H$ for some sequence $g_{1}, \cdots, g_{n}$ in $G$. Now

$$
V_{i}=\bigcap_{t \in W o_{i} H} t^{-1} V t \supset \bigcap_{h \in H} h^{-1} g_{i}^{-1} W g_{i} h
$$

and

$$
\bigcap_{t \in G} t^{-1} V t=\bigcap_{i=1}^{n} V_{i} \supset \bigcap_{h \in H} h^{-1}\left(\bigcap_{i=1}^{n} g_{i}^{-1} W g_{i}\right) h
$$

This proves the lemma.

CoRollary. If in addition to the hypothesis of Lemma 2, $H$ has equal left and right uniformities, then the topologies of $H$ relative to $G$ and $G^{*}$ are equal.

LEMmA 3. Let $G$ be a topological group such that $G=L K$ where $K$ is a compact invariant subgroup and $L$ is a connected subgroup. Suppose that, if $V$ is a neighborhood of the identity relative to $L$, then $V K$ is a neighborhood of the identity in $G$ and for some neighborhood $V_{0}$ of $e$ relative to $L, V_{0} \cup K=e$. Then $G^{*} / K=(G / K)^{*}$.

Proof. Let $U$ be a neighborhood of the identity in $G$ and let $V$ be a symmetric neighborhood of the identity relative to $L$ such that $V \subset U$ and $V^{6} \subset V_{0}$. Let $W$ be a neighborhood of $e$ in $G$ such that $W K \subset V K$. Since $K$ is compact, we can choose a neighborhood $N$ such that $k N \subset W k$ for each $k \in K$. To prove the lemma it is sufficient 
to show that $\bigcap_{g \in G} g N K g^{-1} \subset\left(\bigcap_{l \in L} l U l^{-1}\right) K$ since the second set is a neighborhood of the identity in $G^{*} / K$ by Lemma 2 . Now $\bigcap_{g \in a} g N K g^{-1}$ $=\bigcap_{l \in L, k \in K} l k N K l^{-1} \subset \bigcap_{l \in L} l W K l^{-1} \subset \bigcap_{l \in L} l V K l^{-1}$. We complete the proof by showing

$$
\bigcap_{l \in L} l V K l^{-1}=\left(\bigcap_{l \in L} l V l^{-1}\right) K
$$

Since $L$ is connected, we have $\bigcap_{n=1}^{\infty} \bigcap_{l \in V^{n}} l V K l^{-1}=\bigcap_{l \in L} l V K l^{-1}$. Let $x \in \bigcap_{l \in V} l V K l^{-1}=\bigcap_{l \in V} l V l^{-1} K$. Then for each $l \in V$ we have $x \in l V l^{-1} k$. To see that $k$ does not depend on $l$ let $x=l v l^{-1} k_{1}=h v^{\prime} h^{-1} k_{2}$ where $l$, v, $h, v^{\prime}$ are in $V$ and $k_{1}, k_{2}$ are in $K$. Then, since $V_{0} \cap K=e$ and $V^{6} \subset V_{0}$, it follows that $k_{1}=k_{2}$ and $x \in\left(\bigcap_{l \in V} l l l^{-1}\right) K$. By induction we have $\bigcap_{l \in V^{n}} l V l^{-1} K=\left(\bigcap_{l \in V^{n}} l V l^{-1}\right) K$ and a simple argument now shows that $(\alpha)$ is satisfied.

We will now make use of the following theorem proved by Iwasawa and Yamabe. (See Mem. Amer. Math. Soc., No. 14, 1955; p. 24.)

THEOREM. Let $G$ be a locally compact connected group. There is a neighborhood $N$ of the identity of $G$, a closed local Lie group $L_{0}$ in $N$ and a compact subgroup $K$ such that $N=L_{0} \times K$.

If we let $L$ be the group generated by $L_{0}$, then using the method of the proof of Lemma 3 we obtain the conclusion of that lemma in this case and $G / K$ is a Lie group. To see this let $V$ be a neighborhood of the identity in $L_{0}$ such that $V^{3} \subset L_{0}$. Now assume that $V K$, as a neighborhood of the identity of $G / K$, contains a nontrivial subgroup. Then the inverse image $H$ of this subgroup properly contains $K$ and a simple computation shows that $H \cap V$ is a nontrivial group contained in $L_{0}$ which is a contradiction. We have proved the following.

LEMma 4. If $G$ is a locally compact connected group, then there is a compact invariant subgroup $K$ of $G$ such that $G / K$ is a Lie group and $G^{*} / K=(G / K)^{*}$.

TheOREM 1. If $G$ is a connected Lie group, then $G^{*}$ is a Lie group.

Proof. It is sufficient to prove that $G^{*}$ is locally compact. To do this we use 1.12 , p. 100 of [4]. Let $M$ be the tangent space of $G$ at $e$. For $g \in G$ let $\phi_{g}$ be the inner automorphism determined by $g$ and denote by $d \phi_{g}$ the differential of $\phi_{g}$. Now $d \phi_{g}$ is an isomorphism of $M$ and $\exp d \phi_{0}=\phi_{0} \exp$, where exp is the exponential map of $M$ to $G$. Now let $W$ be a neighborhood of the origin in $M$ such that $\exp \mid W$ is a homeomorphism. There are neighborhoods $U$ and $V$ of $e$ in $G$ such that $V=\exp Z$, where $Z$ is symmetric and convex and 
$g V g^{-1} \subset \exp W$ for $g \in U$. Since $g(\exp Z) g^{-1} \subset \exp W, d \phi_{g}(Z) \subset W$ for $g \in U$. We obtain a sequence of sets $\left\{V_{n}\right\}$ as follows:

$$
\begin{aligned}
& V_{1}=\bigcap_{0 \in U} g V g^{-1}, \\
& V_{n}=\bigcap_{0 \in U} g V_{n-1} g^{-1}=\bigcap_{0 \in U^{n}} g V g^{-1} .
\end{aligned}
$$

Let $F(X)=\bigcap_{g \in U} d \phi_{g}(X)$. We show

$$
\exp F^{n}(Z)=V_{n}
$$

First $\exp F(Z)=\exp \bigcap_{o \in U} d \phi_{o}(Z)=\bigcap_{o \in U} \exp d \phi_{o}(Z)$ since $\exp$ is 1-1 on $d \phi_{g}(Z)$. Thus, $\exp F(Z)=\bigcap_{g \in U} \phi_{g}(\exp Z)=V_{1}$. This proves $(\alpha)$ for $n=1$ and since $V_{1} \subset V$ we have also shown that $F(Z) \subset Z$ and hence $F^{n}(Z) \subset Z$. It follows that exp is 1-1 on $d \phi_{g}\left(F^{n}(Z)\right)$. Now suppose that $(\alpha)$ holds for the positive integer $n$. Then $\exp F^{n+1}(Z)$ $=\exp \bigcap_{\theta \in U} d \phi_{g}\left(F^{n}(Z)\right)=\bigcap_{g \in U} \phi_{\theta}\left(V_{n}\right)=V_{n+1}$ and $(\alpha)$ is proved. Since $Z$ is symmetric and convex $\cap F^{n}(Z)$ is symmetric and convex. Thus, $\exp \cap F^{n}(Z)=\cap V_{n}$ contains a Euclidean neighborhood of $e$. But $\bigcap_{n} V_{n}=\bigcap_{n} \bigcap_{o \in U^{n}} g V g^{-1}=\bigcap_{g \in G} g V g^{-1}$. This proves the theorem.

THEOREM 2. If $G$ is a locally compact connected group, then $G^{*}$ is locally compact.

Proof. By Lemma 4 pick a compact invariant subgroup $K$ such that $G / K$ is a Lie group and $G^{*} / K=(G / K)^{*}$. It follows that $G^{*} / K$ is locally compact by Theorem 1 . By Lemma $1 K$ is a compact subset of $G^{*}$ and by a theorem of Gleason $[3,1.10] G^{*}$ is locally compact.

The following is an example of a locally compact group $G$ such that $G^{*}$ is not locally compact.

ExAmple. Let $A_{i}=Z_{2}$, the discrete two element group, for each integer $i$. Let $H_{0}=\pi_{i} A_{i}$ and let $Z$ be discrete infinite cyclic with generator $z$. Define an operation in $G_{0}=Z \times H_{0}$ as follows, $\left(z^{n}, x\right)\left(z^{m}, y\right)$ $=\left(z^{n+m}, x_{-m} y\right)$ where the $i$ th coordinate of $x_{-m}$ is the $(i-m)$ th coordinate of $x$. Thus $G_{0}$ is a semidirect product [5]. It is easy to see that $G_{0}$ is a locally compact group with this operation and the usual product topology. Let $G_{i}=G_{0}$ and $H_{i}=\{e\} \times H_{0}$ for $i=1,2, \cdots$. Let $H=\prod H_{i}$ and $G=\prod G_{i}$ with coordinatewise operation, the usual product topology on $H$ and $H$ open in $G$. Thus, $G$ is a locally compact group and $H$ is a compact open subgroup of $G$. Let $V_{i}$ be a proper neighborhood of $e$ in $H_{i}$. If $U_{n}=V_{1} \times \cdots \times V_{n} \times\left(\prod_{i>n} H_{i}\right)$, then $\bigcap_{t \in G} t U_{n} t^{-1}=\{e\} \times \cdots \times\{e\} \times\left(\prod_{i>n} H_{i}\right)$. Since $\left\{U_{n}\right\}$ is a neighborhood base at the identity of $G$, it follows that $G^{*}$ is not locally compact. 
A more general construction than that above is obtained by letting $F$ be a compact infinite group which admits an expansive automorphism $\phi$. Let $H$ be the discrete group generated by $\phi$. Now replace $G_{0}$ by the semidirect product $F(S H$ in the above example. The existence of expansive automorphisms on certain compact groups has been established by R. F. Williams [3], T. S. Wu [11], M. Eisenberg [2] and F. Reddy [8].

By a one parameter subgroup in a group $G$ we mean the image $\phi(R)$ of the reals under a continuous homomorphism $\phi: R \rightarrow G$.

THEOREM 3. If $G$ is a connected locally compact group and $\phi(R)$ is a one parameter subgroup of $G^{*}$ which is normal, then $\phi(R)$ is contained in the center of $G$.

Proof. By Theorem $2 G^{*}$ is locally compact. Thus $\phi$ is a topological isomorphism or $\bar{\phi}(R)$ is compact in $G^{*}[7$, p. 102]. In case $\bar{\phi}(R)$ is compact in $G^{*}$ it is also compact in $G$ and hence contained in the center of $G[5,26.10]$. Now suppose that $\phi$ is an isomorphism. Pick a neighborhood $V$ of $e$ in $G$ such that $\bigcap_{g \in G} g V g^{-1} \cap \phi(R)=(-\epsilon, \epsilon)$. Let $\alpha$ be in $(0, \epsilon)$ and define a sequence in $(-\epsilon, \epsilon)$ as follows, $\alpha_{1}=g \alpha g^{-1}$, $\alpha_{n}=g \alpha_{n-1} g^{-1}$ where $g$ is any element of $G$. The sequence $\left\{\alpha_{n}\right\}$ is monotone. Let $\beta=\lim \alpha_{n}$. It follows that $g \beta g^{-1}=\beta$. We can assume that $\beta$ is nonzero since, if $\left\{\alpha_{n}\right\}$ is decreasing, on replacing $g$ by $g^{-1}$ we get an increasing sequence and its limit will also commute with $g$. It is easy to show that if $g$ commutes with a nonzero real number $\beta$ then it commutes with every rational multiple of $\beta$ hence with every real number. Thus, $\phi(R)$ is contained in the center of $G$.

As an immediate consequence of Theorem 3 we have.

COROLlaRy 1. If $G$ is a connected locally compact group with equal left and right uniformities which contains a one parameter group as a normal subgroup then this subgroup is in the center of $G$.

COROLlARY 2. If $G$ is a connected locally compact group which contains a one parameter normal subgroup $\phi(R)$ such that $G / \phi(R)$ is compact, then $\phi(R)$ is contained in the center of $G$.

Proof. If $\phi(R)$ is compact, then the conclusion follows from [5, 26.10]. Otherwise $\phi$ is an isomorphism [7, p. 102] and by the Corollary of Lemma $2 \phi(R)$ is a one parameter subgroup of $G^{*}$. The corollary now follows from the theorem.

\section{REFERENCES}

1. Jean Braconnier, Sur les groupes topologiques localement compacts, J. Math. Pures Appl. 27 (1948), 1-85. 
2. M. Eisenberg, Doctoral Dissertation, Wesleyan Univ., Middletown, Conn., 1965.

3. A. M. Gleason, The structure of locally compact groups, Duke Math. J. 18 (1951), 85-104.

4. S. Helgason, Differential geometry and symmetric spaces, Academic Press, New York, 1962.

5. E. Hewitt and K. A. Ross, Abstract harmonic analysis, Academic Press, New York, 1963.

6. K. Iwasawa, On some types of topological groups, Ann. of Math. 50 (1949), 507558.

7. D. Montgomery and L. Zippin, Topological transformation groups, Interscience, New York, 1955.

8. F. Reddy, Expansive homeomorphisms (to appear).

9. R. F. Williams, $A$ note on unstable homeomorphisms, Proc. Amer. Math. Soc. 6 (1955), 308-309.

10. T. S. Wu, Left almost periodicity does not imply right almost periodicity, Bull. Amer. Math. Soc. 72 (1966), 314-316.

11. - Expansive automorphisms on groups, Math. Scand. 18 (1966), 23-24.

UNIVERSITY OF Miami AND

Case Institute of Technology 